\title{
Zur Geschichte der Basler Medizinischen Fakultät im ersten Drittel dieses Jahrhunderts*
}

\author{
Von Friedrich Rintelen
}

Aus der Geschichte der Basler Medizinischen Fakultät im ersten, politisch allgemein so bedrängenden Drittel unseres Jahrhunderts seien einige Aspekte hervorgehoben, die man problematisch nennen kann.

Das griechische $\pi \varrho o ́ ~ \beta \lambda \eta \mu \alpha$ bedeutet etwas, das Menschen und Menschengemeinschaften als ein oft leidiges Hindernis gleichsam vom Schicksal vorgeworfen ist; oft sind es selbstbereitete Schwernisse durch Tun und Lassen der Beteiligten.

Es schien richtiger, auf Laudationen eher zu verzichten, obschon sich dazu manche Gelegenheit böte. Man hätte etwa über die drei großen Roberte berichten können: Robert Bing, den großartigen Didakten der Neurologie, Robert Doerr, den originellen Forscher besonders im Bereich der Virologie, Robert Rössle, den hervorragenden Vertreter der allgemeinen Pathologie, die ungewöhnlich weit wirkenden wissenschaftlichen und didaktischen Leistungen dieser Männer. Auch über den so aktiven Alfred Gigon, nicht nur verdient um die erfreuliche Entwicklung der medizinischen Poliklinik, sondern auch um die Gründung der schweizerischen Akademie der medizinischen Wissenschaften und um entscheidende Anregung zur Errichtung des Tropeninstitutes in Basel; über Hans Staub, den so diametral anders veranlagten Mann, dem man später im Bereich der inneren Medizin sauberstes pharmakologisch fundiertes Denken, Urteilen und Handeln verdankt.

Auch von «Alt-Baslern» hätte die Rede sein können; etwa von John Staehelin, dem der entscheidende Wandel der Irrenanstalt «Friedmatt» zur offenen psychiatrischen Klinik gelang, von dem Arzt, der seine reichen und vielfältigen Gaben in der Atmosphäre baslerischer Kultur zu entfalten wußte. Oder von dem Pathologen Andreas Werthemann, dem heiteren zweimaligen Rektor der Hochschule, und seinen Verdiensten um das Gelingen der 500-Jahr-Feier von 1960, von diesem Menschen erhellender Güte, der als Freund von Hans Münch auch im Musikleben der Stadt eine führende Rolle gespielt hat; ein Wirken, das freilich mehr ins zweite Drittel des Jahrhunderts gehört. Allerdings wären auch bei solchen Männern ge-

* Nach einer «Grundsatzvorlesung» vor Semesterschluß, 20. Februar 1981.

Gesnerus 1/2 (1981) 
wisse Grenzen nicht zu übersehen, die ihnen in der Problematik ihres So-seins durch angeborene Konstitution und erworbene Kondition gesetzt waren.

Nützlicher mochte es sein, vorwiegend vom generell Problematischen in der Existenz der Fakultät, der Menschen, die sie vorübergehend repräsentieren, zu sprechen, Tatsachen und Meinungen anzuführen, die freilich für Fakultät und Behörden nicht nur schmeichelhaft gewesen sind. Um ein beschaulich-erbauliches Betrachten einer neueren historischen Antiquität sollte es nicht gehen.

1900 gab es an der Fakultät acht Lehrstühle für ordentlich-öffentliche Professoren. Das o. oe. vor dem Professorentitel ist für viele lange Zeit eine gepflegte Beigabe gewesen. Zu den vier Ordinariaten des damaligen, im Prinzip noch gültigen Universitätsgesetzes von 1866, für Anatomie-Physiologie, Pathologie, internistische und chirurgische Klinik, waren vier weitere gekommen: besondere Lehrstühle - um dies heute ungern gehörte, aber seßhafte Substantiv zu verwenden - 1873 für Physiologie, 1875 für Psychiatrie, 1887 für Gynäkologie und 1892 für Hygiene. Für andere im Unterricht wichtige Fächer gab es lediglich Lehraufträge mit möglichem Ordinariat; die waren billiger. Nun, auf 7 der 8 Lehrstühle saßen damals deutsche Professoren. Kein Zweifel, Persönlichkeiten wie etwa Friedrich Müller, später führender Internist in München, Ernst Bumm, nachmals kaiserlicher Accoucheur in Berlin, oder der autoritäre Didakt, der Pathologe Eduard Kaufmann, um nur drei Hervorragende zu nennen, haben fruchtbare Anregung gebracht und Wesentliches zum Ausbau und Ansehen der Fakultät beigetragen. Charakteristischerweise sind sie in Basel nur ausnahmsweise heimisch geworden; selten haben sie eine wirkliche Beziehung zur Stadt gefunden, selbst wenn sie bis zur Emeritierung oder bis zum Tode hierblieben, wie der Psychiater Wille, der Physiologe Metzner und der Anatom Kollmann. Typisch, daß der Gynäkologe v. Herff, obwohl mit einer Schweizerin verheiratet, sich hier fremd fühlte und fast bis zuletzt vergeblich auf einen Ruf an eine deutsche Hochschule wartete. Der hier beliebte Internist Gerhardt schreibt, als er einen Ruf nach Würzburg bekommt: «So glücklich meine Familie und ich in der Schweiz waren, so fühlte ich mich doch als Zugewanderter und blieb im Herzen ein Deutscher.» Das galt für viele. Wenn deutsche Ordinarien hierblieben, so bis 1933 nur wenige - etwa Karl Spiro, Ordinarius für physiologische Chemie -, weil sie sich gerade hier, in einer demokratischen und kritischen Atmosphäre, wohl fühlten und gelegentlich auch einen Ruf ins Reich ablehnten. Wer hierblieb, tat es meist, weil er in Deutschland fachlich zuwenig konkurrenzfähig schien oder aber 
sich wegen menschlicher Eigenwilligkeit nicht empfahl, wie der Balte Gustav v. Bunge, der in seinen Basler Jahren zum fanatischen Abstinenzprediger geworden ist. Bei anderen war es mangelnde «Gesinnungstüchtigkeit», ein ebenso bezeichnendes wie bedenkliches Wort, das bei deutschen Politikern in disqualifizierendem Gebrauch war. Deutschen war das Basler Ordinariat ein erwünschtes Sprungbrett zu vermeintlich wertigeren fachlichen Aufgaben in Deutschland. Sie blieben meist nur wenige Jahre, oft so kurz, daß die Regierung bei Berufungen die bescheidene Zusage verlangte, mindestens vier Jahre in Basel zu bleiben. Nicht alle haben sich an das gegebene Wort gehalten - der Internist Fritz Voigt blieb ganze acht Monate! Der häufige Chefwechsel war besonders in den Kliniken des Bürgerspitals in erster Linie für Assistenten und Patienten unerfreulich. Ich meine, es sei auch nicht zu übersehen, daß für die ungute politische Entwicklung in Deutschland vor und nach dem Ersten Weltkrieg ein solches Springen und Klettern von niedriger zu höher bewerteten Lehrstühlen - auch innerhalb des Reiches - mitverantwortlich war. Ähnliche Sprünge gab es damals auch bei Generälen und höheren Beamten. Wie viele Professoren, so fühlten auch die sich nicht eigentlich als verantwortliche Bürger eines Gemeinwesens, blieben ungeschult, unerfahren in selbständigem politischem Denken und Handeln, waren zunächst schlechthin Deutsche. Nicht wenige konnten derart in den Sog nationaler Hegemonieansprüche von Politikern und des bis 1918 dominierenden großen Generalstabes geraten. Die Nation verdrängte das Individuum. Heute stehen oft wissenschaftliches Interesse und fachliche Belastung einem politischen Engagement entgegen.

Wer zwischen 1900 und 1933 nach Basel kam, war kein politisch Verfolgter, kein Refugiant wie einst Karl Gustav Jung, der Reorganisator der medizinischen Fakultät um die Zeit der Kantonstrennung, wie Leberecht de Wette, der Theologe, oder der Germanist Wilhelm Wackernagel. Die suchten hier Asyl und fanden in Basel bleibenden Kontakt mit einer neuen Heimat.

Obschon Basel heute zu einem fast polyglotten schweizerischen «grüetzi»Mélange geworden ist, - die Stadt, ihr Kern, ist für Fremde auch heute noch ein Boden, auf dem man nicht leicht anwächst, trotz unverkennbaren Assimilationskräften. Manchen hat er hier das Heimisch-Werden erschwert, dieser früher freilich viel eindeutigere genius loci. Er ist bei «Altbaslern» jedenfalls mitgekennzeichnet durch kritischen Skeptizismus des geborenen Kaufmanns, der zum Wagnis weniger geneigt und auf das Maßvolle bedacht ist. Typisch die gelegentlich fast süchtige Neigung zur Kritik, die Hemmung 
zu loben, eher Sinn für die Schwächen des Mitmenschen, freilich auch für die eigenen; die übliche Vorsicht, sich womöglich nicht tiefer in die Seele blicken zu lassen. Große Sparsamkeit, mit natürlichem Erwerbssinn gepaart, gehören dazu; bei Behörden gelegentlich Anlaß zu einem für die Universität beschwerlichen Zweckpessimismus hinsichtlich der Finanzlage des kleinen Stadtkantons. Freilich ist diesem génie propre auch ausgesprochener Sinn für das Bewahren und Fördern geistiger Werte eigen, für ein nobile officium zu großzügiger Wohltätigkeit, eher in der Stille, bei Abneigung gegen jedes Pathos. Förderung und Hemmnis, befreiende Weite und kleinliche Enge liegen hier nahe beisammen, auch heute noch zu überraschenden Wirkungen bereit. Auch das schließt Problematik ein. Einzigartigkeit des geographischen Raumes gehört dazu: der fast jäh sich nach Norden wendende Strom, dort wo Jura, Vogesen und Schwarzwald sich so nahe kommen, ohne sich zu berühren; die Weite der offenen Burgundischen Pforte nach Südwesten, nach Frankreich, sie prägen die Stadt; so wie die nicht nur orographische Grenze des Jurakammes gegen die übrige Schweiz, die ein unübersehbares Abseitsstehen Basels in der Eidgenossenschaft miterklärt. Diese Lage ist aber auch charakterisiert durch ein nicht allen zuträgliches, irritatives Klima. So konnte der Internist Friedrich Müller bei seinem Amtsantritt in Basel sagen: «Beim Auspacken befiel mich eine merkwürdige Müdigkeit, die ich nie zuvor gekannt habe. Ich wurde aufgeklärt, daß dies eine Folge des Föhns sei, der sich in Basel höchst unangenehm bemerkbar mache.» Freilich ist es kein eigentlicher Föhn, sondern der Durchgang von Wetterfronten mit ihren schnell von Westen ziehenden Wolken. Jahre später hat der Pathologe Rössle, der es hier immerhin 7 Jahre, Großes leistend, ausgehalten hat, nach Berlin berufen, erklärt, es sei höchste Zeit, von Basel fortzukommen, damit er wieder arbeiten könne!

Aus der übrigen Schweiz nach Basel Berufene fühlten sich hier manchmal in einer beengenden Diaspora; sie wurden wohl zuwenig akklamiert, sehnten sich nach größerer Resonanz. Auch deshalb verlor man den Pathologen Hedinger, den Ophthalmologen Vogt und den Dermatologen Bloch nach Zürich, den Chirurgen de Quervain nach Bern. Es lockten ein größeres Krankengut, mehr Studenten, höhere Einkünfte, anscheinend leichtere Lebensbedingungen.

Für Schweizer Mediziner war ein Ruf an eine deutsche Universität eine Ausnahme; 1872 etwa für den älteren Wilhelm His, den Anatomen, nach Leipzig; das kümmerliche anatomische Kabinett im unteren Kollegium am Rheinsprung konnte ihn begreiflicherweise nicht halten. 1906 holte man 
seinen Sohn, den Internisten gleichen Namens, nach Göttingen, schließlich nach Berlin. Er war großenteils in Leipzig aufgewachsen, wurde schließlich preussischer Staatsangehöriger und fühlte sich offenbar weitgehend als solcher; er hat es im Ersten Weltkrieg bis zum beratenden Internisten einer Armee gebracht. Von seinen Kriegserlebnissen vor Verdun, im Kaukasus wie im nahen Osten hat er in seinem Buch «Die Front der Ärzte» mit erstaunlicher Begeisterung berichtet.

Der Psychiater Rüdin, gebürtiger St. Galler, bisher an der genealogischen Abteilung der deutschen Forschungsanstalt für Psychiatrie in München, war 1925 als Nachfolger Gustav Wolffs nach Basel gekommen. Er blieb nur zwei Jahre, um dann, einem Rufe folgend, nach München zurückzukehren; es gehe um die Verwirklichung seines Lebenswerkes. Dort ist Rüdin, vielleicht als Genealoge dafür eher disponiert, offensichtlich nationalsozialistischem Ungeist erlegen. Er hat die Reihe «Erblehre und Rassenhygiene im völkischen Staat» ediert und ist für das folgenschwere «Gesetz zur Verhütung erbkranken Nachwuchses» entscheidend mitverantwortlich; gelegentlich waren damals auch Schweizer infizierbar.

Um die Jahrhundertwende war an der Fakultät Albrecht Burckhardt, dem man eine ausgezeichnete Geschichte der Basler medizinischen Fakultät von 1460 bis 1900 verdankt, unter den Ordinarien als Schweizer und Basler die große Ausnahme. Wenn für das neugeschaffene Fach der Hygiene die Wahl auf einen Basler wohl fallen mußte, so hauptsächlich deswegen, weil mit diesem Ordinariat damals die Leitung des schulärztlichen Dienstes verbunden war. Dafür hätte sich kaum ein Auswärtiger geeignet. Burckhardt ist in administrativen Belangen, zumal in Berufungsfragen, lange zur richtunggebenden Persönlichkeit in der Fakultät geworden.

Warum gab es, besonders im ersten Dezennium dieses Jahrhunderts, so viele deutsche Ordinarien an der Fakultät? Von 1900 bis 1933 waren es im ganzen 23. In Basel waren übrigens 1910 von 130000 Einwohnern 52000 , also $38 \%$, Ausländer, und zwar vorwiegend Deutsche! - Galten bei den Behörden, bei Kuratel und Erziehungsrat, die Einheimischen weniger? Allerdings, wer hier aufgewachsen war, den kannte man besser, auch seine Schwächen, begegnete ihm mit schärferer Kritik; intrigierende Einflüsse auf dem langen Weg des Berufungsverfahrens über Fakultät-Kuratel-Erziehungsrat zur Regierung waren wahrscheinlicher. Entscheidender war aber wohl die Tatsache, daß es am eigenen schweizerischen Nachwuchs fehlte. Besonders in den Grundlagenfächern der Medizin gab es zuwenig und zu schlecht bezahlte Assistentenstellen. Das ist jahrzehntelang so geblieben. 
Noch 1934 hat August Ruegg, begeisternder Lehrer am Gymnasium und späterer Extraordinarius für iberoromanische Literatur, in der Kuratel gemahnt, man müsse Nachwuchskräfte entsprechend bezahlen; nur wenige könnten sich eine wissenschaftliche Tätigkeit in schlecht honorierten Assistentenstellen leisten. Das galt allgemein für die Hochschule. 1906, anläßlich einer geplanten Studienreform, wurde von der Fakultät ein obligates Assistentenjahr nach dem Staatsexamen mit der Begründung abgelehnt, es gäbe nicht genügend Assistentenstellen. Erstaunlich, daß trotzdem zwischen 1900 und 1913 recht viele Habilitationen zustande gekommen sind; es waren überwiegend Kliniker. Das hat sich auf Berufungen aber erst viel später ausgewirkt. Die Habilitation gehörte und gehört übrigens zu den wenigen akademischen Rechten der Fakultät. Allerdings war die Stellung der Privatdozenten damals schwach, viel schwächer als heute; kaum daß sie gelegentlich angehört wurden; ihre Forschungsmöglichkeiten waren spärlich; nur wenige kamen zum Dozieren, trotz einem enormen Wochenstundenangebot; um 1900 waren es 65 Stunden - selbst samstags von 7 bis 1 Uhr und von 2 bis 5 Uhr wurde gelesen -. Gemeinschaftsvorlesungen gab es außer einem Ordinarienkränzchen noch nicht. Die Zahl der Medizinstudenten war klein; im Sommersemester 1900 waren es 139. Eine einzige Medizinstudentin war dabei. Der Regierungsrat hatte 1890 die Zulassung der Frau zum Studium geregelt. Aber noch am Dies 1891 wetterte der Rektor, ausgerechnet der Gynäkologe Fehling, damals wohl noch mit dem inneren Einverständnis vieler, gegen das Frauenstudium: «die Frau habe Gattin, Hausfrau und Erzieherin der Jugend zu sein»; basta! Heute sind 25\% der MedizinStudierenden und $17 \%$ der Gesamt-Ärzteschaft Frauen. Wie die Besoldungssituation noch 1940 war, zeigt das Bemühen des späteren Chefs der medizinischen Klinik Hans Staub, damals Vorsteher des pharmakologischen Instituts. Er versuchte vergeblich, für seinen einzigen bewährten älteren Assistenten eine bessere Besoldung zu erwirken als die eines Straßenbahners - wie er sagte - . Der Sozialdemokrat Dr. Fritz Hauser, Vorsteher des Erziehungsdepartementes, für die Anliegen der Universität sonst so aufgeschlossen, fand, die Assistenzen der Universitätsanstalten seien keine Lebensstellen; man lerne da viel, was im späteren Berufe nützlich sei. Er übersah, daß Assistenten an Instituten für medizinische Grundlagen sich nur profilieren konnten, wenn sie längere Zeit blieben; dann aber hatten sie es schwer, aus solch einer, materiell gesehen, eher brotlosen Kunst den Weg in die Praxis zu finden. Auch an den Kliniken war die Zahl der karg besoldeten Assistenten bescheiden; sie reichte wohl für sorgfältige Betreu- 
ung der Patienten, kaum aber auch für wissenschaftliche Arbeit. Deswegen ist Friedrich Müllers Aussage hochmütig und ungerecht, wenn er fand: «Die Schweizer Assistenten waren zuverlässig und gut ausgebildet, aber frei von allen wissenschaftlichen Interessen» - es fehlte die Zeit, nicht das Interesse-. Die Behörden haben da oft am falschen Ort gespart; es ist deshalb nicht erstaunlich, daß es bis in die späteren dreißiger Jahre bei manchen Berufungen hieß, qualifizierte Schweizer seien nicht vorhanden.

Problematisch für das Prosperieren der Fakultät waren lange Zeit auch die eher knappen Besoldungen, besonders ungenügende Pensionierungsbedingungen für die Ordinarien. Durch Wegberufungsdrohungen wußten sich freilich einzelne ihre Remuneration erheblich zu verbessern. Allerdings, als dann im Juni 1910 die Universität ihr 450jähriges Jubiläum beging, spendete die freiwillige akademische Gesellschaft 345000 Franken! Oft hat diese Vereinigung, damals unter der Führung des Ratsherren und Juristen Andreas Heusler, nach der Kantonstrennung von 1835 das Fortbestehen der Hochschule überhaupt möglich gemacht. Die Gründung einer freilich noch bescheidenen Witwen- und Waisenkasse kam zustande, aber nur für Lehrstuhlinhaber. Sie hat bei Berufungsverhandlungen manchmal eine wesentliche Rolle gespielt. Der damalige Vorsteher der Gesellschaft, der spätere Korps-Kdt. Isaak Iselin, sagte in seiner knappen Ansprache in der alten Aula: «Freunde der Universität aus allen Kreisen haben sich zusammengetan, bei ihren Mitgliedern angeklopft und ihnen in Erinnerung gerufen, was der Basler seiner Universität schuldig ist.» Es gab damals noch ein deutliches Einssein weiter Teile der Stadt mit der Hochschule.

Zu einem tristen Kapitel: In Berufungsfragen hat sich eine in der Schweiz gleichsam endemische, freilich selten lauter artikulierte Judenfeindlichkeit als besondere Form der Xenophobie in erstaunlicher Unbefangenheit bemerkbar gemacht. Über die weitverzweigten und tiefreichenden Wurzeln eines solchen sogenannten Antisemitismus ist viel geschrieben worden. Jean-Paul Sartre hat in seinen 1946 erschienenen «Réflexions sur la question Juive» in harter Formulierung gesagt: «L'antisémite reconnaît volontiers que le Juif est intelligent et travailleur, mais il a mis ses qualités en parentaise. Plus le Juif aura des vertus, plus il sera dangereux. L'antisémite se considère comme un médiocre. La phrase, je haïs les Juifs est de celles qu'on prononce en groupe; en la prononçant on se rattache à une communauté, celle des médiocres.» In welchem Ausmaß sich solche Ansichten über jüdische Mitmenschen auch in der Fakultät bemerkbar machten, ist schwer abzuschätzen. Man darf immerhin feststellen, daß in Basel oder in der 
Schweiz aufgewachsene Juden wie der Dermatologe Bloch oder der Gerichtsmediziner Schönberg durchaus akzeptiert wurden, daß Karl Spiro nicht nur geschätzt, sondern verehrt wurde; Robert Bing hatte viel eher seines von den Internisten beanspruchten Faches, der Neurologie wegen Schwierigkeiten als wegen seiner jüdischen Abstammung, auf die er stolz war. Aber man muß wissen, daß in der Kuratel antisemitische Töne immer wieder zu hören waren oder ankamen; dort saßen gute Basler. Als es 1922 um Hedingers Nachfolge für die Pathologie ging, ließ man das in Deutschland und Österreich von der Zürcher Fakultät für den gleichen Lehrstuhl angeforderte Gutachten kommen. Dort hieß es vom Genfer Ordinarius Askanazy, er sei hervorragend, habe aber ein ausgeprägtes, nicht angenehmes jüdisches Wesen. Der Pathologe Otto Lubarsch schrieb aus Berlin, er selbst stehe politisch auf dem Boden der Rechtsparteien, die «den unheilvollen, übermächtigen Einfluß des Judentums scharf bekämpfen». Der angesehene Pathologe Aschoff in Freiburg meinte gewunden von einem Wiener Kollegen: «Er ist ausgesprochener Jude; ich persönlich und Viele mit mir werden darüber hinwegsehen, aber ich kenne die Zürcher Studentenschaft nicht.» Aus Wien hörte man ähnliches; von einem fachlich ausgezeichneten Kandidaten hieß es: «Er ist zwar getaufter Jude, gehört aber zur deutschen Partei», das heißt gegen die Slawen und Sozialdemokraten; man habe bei ihm keine sogenannten jüdischen Eigenschaften kennengelernt! Das Terrain, zunächst für den Mord an Walther Rathenau, mit Stresemann bekannt als Vertreter der deutschen Verständigungs- und Erfüllungspolitik nach Versailles, war bereitet. In Kreisen der Universität, gerade auch bei den Medizinern, konnten die Nationalsozialisten in Deutschland wie in Österreich bald mit einiger Gefolgschaft rechnen. Erstaunlich, daß man aus antijüdischen Äußerungen Schweizern gegenüber kein Hehl machte; offenbar glaubte man hier oft mit ähnlicher Einstellung rechnen zu können; wohl nicht ganz zu Unrecht. In der Expertenkommission in Basel hieß es schließlich, man möchte einem Nichtjuden den Vorzug geben; die Wahl fiel auf Robert Rössle. Als für den Dermatologen Bloch 1916 «zwei erstklassige christliche Kandidaten» - wie es wörtlich hieß - nicht in Frage kamen, glaubte die Expertenkommission, «nicht davor zurückschrecken zu sollen, einen jüdischen Kandidaten vorzuschlagen»; es war der ungewöhnlich kultivierte Hamburger Dermatologe Felix Lewandowsky. Lange vor dem Aufkommen des Nationalsozialismus ist solches und ähnliches zu hören gewesen. 1906 galt es, den Internisten Wilhelm His zu ersetzen. Von einem qualifizierten Kandidaten schreibt Albrecht Burckhardt, der jeweilen Papabiles im 
Auftrag von Fakultät und Kuratel an ihrem Wirkungsort zu besuchen pflegte: «Die Befürchtung, er sei vielleicht Israelit, ist unbegründet.» Das erstaunliche Alibi lautet, er sei «mit einer katholischen Pariserin aus höheren Militärkreisen verheiratet». Von einem anderen hieß es, er verfüge über ein hervorragendes Lehrtalent, sei «unstreitig bedeutender als seine Mitkandidaten, aber jüdischen Ursprungs und mit einer Jüdin verheiratet, allerdings schon als Kind umgetauft». Aber die «Berufung eines Israeliten schließe eben doch gewisse Gefahren in sich und führe möglicherweise zu Unzuträglichkeiten». Beide Kandidaten sind nicht gewählt worden. Es galten offenbar erstaunliche Kriterien!

Im Wintersemester 1933/34 studieren 581 Mediziner in Basel, mehr Ausländer als Schweizer, vor allem jüdische Flüchtlinge aus Deutschland. Es sind so viele, daß die hiesige jüdische Gemeinde bittet, nicht zu viele aufzunehmen; sie befürchtete antisemitische Demonstrationen. Ein Bundesratsbeschluß vom April 1933 empfiehlt den Kantonen Maßnahmen gegen Einbürgerungstendenzen und Erwerbstätigkeit von Ausländern. Die Zuwanderung Mittelloser solle verhindert werden. In Basel müssen jüdische Studenten einen Revers unterschreiben, laut welchem Erwerb und Einbürgerung ausgeschlossen sind. Man war sich in dieser peinlich-kleinlichen Haltung offenbar noch nicht bewußt, in wie tragischer Weise sich die Situation im weiten nationalsozialistischen Machtbereich sehr bald entwikkeln sollte. Eigene Sorgen standen auch in der Fakultät im Vordergrund. Angesichts des Andrangs ausländischer, fast ausschließlich jüdischer Mediziner, der eigentlich ohne besondere Anstrengungen zu verkraften gewesen wäre, machte sich oft eine wenig verständnisvolle, national orientierte, letztlich unmenschlich wirkende Mentalität bemerkbar. So wird ausländischen Studenten, die im Examen durchfallen, vom Polizeidepartement ein weiterer Aufenthalt in Basel und in der Schweiz verboten, und die Fakultät stimmt dem zu!

Daß man an Basels Hochschule die politische Entwicklung in Deutschland mehrheitlich nicht allzu tragisch nahm, mag auch die Tatsache illustrieren, daß sich Ordinarien und Extraordinarien der Fakultät 1934 für die Münchner medizinische Wochenschrift photographieren ließen. Peinlicher war es, daß sich die Regenz 1936 und 1937 den geplanten Besuch an Jubiläumsfeiern in Heidelberg und Göttingen von den oberen Behörden verbieten lassen mußte, weil dies, wie es mit Recht hieß, «unvermeidbar mit nationalsozialistischem Gepränge verbunden sei». Allerdings gab es in Basel 1935 neben Männern wie Karl Barth und Edgar Bonjour auch unter den 
deutschen Hochschullehrern Dozenten, die die Situation im Hitler-Reich mit kritischer Sorge oder laut werdender Erbitterung betrachteten. Offenbar haben sich damals auch nur $4 \%$ der in der Schweiz ansässigen Deutschen deutlich zum Nationalsozialismus bekannt. Aber nicht ganz wenige Mitglieder von Regenz und Fakultät haben lange die Gefährlichkeit der Entwicklung in Deutschland unterschätzt; man war allzu fachlich orientiert und konnte sich offenbar schwer vorstellen, daß Kollegen im Dritten Reich einen unguten Weg gingen.

Nur ausnahmsweise aber waren deutsche Dozenten wie der Pathologe Werner Gerlach eigentliche Propagandisten des nationalsozialistischen Systems. Es ist schwer verständlich, wie dieser geschickte Mann in Regenz und Fakultät in unangebrachter, naiver Kollegialität immer wieder in Schutz genommen worden ist. Politische Weitsicht und Fingerspitzengefühl fehlten gelegentlich. In den Fakultätsakten steht kein Wort über einen Fall Gerlach. Auch im Bericht des Rektors der Jahre 1935 und 1936 wird die Angelegenheit verschwiegen, weil sie, als politisch betrachtet, die Universität offenbar nichts anging. Aber gerade daß man sich in der Universität damals um die Machenschaften Gerlachs offiziell nicht kümmerte, hat in Basels Öffentlichkeit dem Ansehen von Universität und Fakultät geschadet. Das gerade in solch kritischer Zeit notwendige Mittragen der Hochschule durch die Bevölkerung der Stadt, das später in harmloser Situation an der 500-Jahr-Feier 1960 wieder so eindrücklich zutage getreten ist, schien gefährdet. Was war geschehen?

Gerlach hatte im Oktober 1935 in Ueberlingen, wo auch der «Gauleiter Schweiz» Gustloff wirkte, die deutsche Studentenschaft in der Schweiz in nationalsozialistischem Sinne de facto subversiv indoktriniert, hatte beim Rektorat der Töchterschule gegen die «ekelhafte und verlogene Hetze der Emigrantenklicke» - wie er sich ausdrückte - protestiert, eine nicht Hitlerkonforme Assistentin in Deutschland effektiv denunziert und außerdem in seinem Institut, das er an sich als beliebter Dozent und geschickter Organisator führte, seine deutschen Mitarbeiter kraß begünstigt. Im Großen Rat hatte der Kommunist Meili interpelliert. Die geforderte disziplinarische Untersuchung durch die zuständige Kommission kam auf Grund der Zeugenaussagen aus Fakultät und Institut zum Schluß, es sei nicht verboten, eine nationalsozialistische Gesinnung zu haben; an Gerlachs Institutsführung sei nichts zu bemängeln. Aber die Kuratel beschloß trotzdem durch Stichentscheid ihres mutigen und klarsehenden Präsidenten, des Ständerates Ernst Thalmann, über den zustimmenden Erziehungsrat der Regierung 
Gerlachs disziplinarische Entlassung zu beantragen. Gerlach ist ohne Entschädigung entlassen worden. Aber der selbstbewußte Herr gelangte an das Appellationsgericht. Der Entlassungsbeschluß des Regierungsrates wurde durch das Appellationsgericht auf Grund des Universitätsgesetzes aufgehoben, wonach die Anstellung von Universitätslehrern «ohne Rücksicht auf deren politische und religiöse Überzeugung erfolge». Darüber war Mottas politisches Departement in Bern, das möglichst Friktionen mit dem Dritten Reich vermied, wahrscheinlich froh. Gerlach, wieder in sein Amt eingesetzt, ist in Institut und Fakultät mit geradezu demonstrativer Freundlichkeit empfangen worden. Weitherum in der Stadt aber war man entrüstet; nicht nur die «Linke» sprach von einem Skandal. Glücklicherweise ist Gerlach von der deutschen Regierung kurz nach dem Entscheid des Appellationsgerichtes abberufen worden; am 1.April 1937 hat er Basel verlassen. Erleichtert schrieb Hauser an den Kuratel-Präsidenten: «Die Berufung Gerlachs enthebt mich der Aufgabe, weitere Schritte gegen diesen Dozenten zu unternehmen.» In Fakultät und Regenz, auch in manchen Kreisen des intellektuellen Basel hatte man die Gefährlichkeit nationalsozialistischer Propaganda zum Teil noch nicht erfaßt. Diese Einsicht kam später.

Auch im fachlichen Bereich war gelegentlich eine gewisse Horizontenge auffallend. Eher zur älteren Generation Gehörende hatten offenbar hie und da Mühe, den Bedürfnissen der Zeit nicht mehr entsprechende konservativtraditionalistische Auffassungen zu revidieren; einzelne glaubten vielleicht, es ihrer Autorität schuldig zu sein, am einmal als richtig Anerkannten festhalten zu müssen, auch wenn sich wandelnde Bedingungen für Neuerungen sprachen. Das ist wohl mehr oder weniger eine physiologische Erscheinung. Damals haben Maßgebliche sich lange etwa gegen Psychologie, Psychohygiene und psychoanalytisches Denken und Lehren als mögliche Ergänzungen einer prophylaktischen und therapeutischen Medizin gesträubt. Kleinlich, ja überheblich lange hat man gezögert, die Zahnheilkunde als einer medizinischen Fakultät würdiges Fach anzuerkennen. Von einer Leidensgeschichte des Zahnärztlichen Instituts ist geschrieben worden. Es hat auch lange gedauert, bis man mehrheitlich einsah, daß Lateinkenntnisse, so horizontweitend und sprachlich disziplinierend sie sind, für das Werden eines Mediziners zum mitmenschlichen Arzt keine conditio sine qua non darstellen müssen, daß ein kurzes Nachholen einer Lateinmatur an der Universität Würde und Wert dieser Sprache eher abträglich ist. Wahre Bildung, deren der Arzt so sehr bedarf, ist an Mittelschulen durch geeignete Lehrer auch in anderen Fächern zu vermitteln. 
Was ich hier darzulegen versuchte, sind einige Feststellungen, die beim Überblicken des vergangenen Zeitabschnittes im Leben der Fakultät nicht übersehen werden durften und die man bei Gelegenheit nicht vergessen sollte. Es gab große Leistungen nicht weniger, zumal im fachlichen Bereich, in Forschung, Lehre und Dienstleistung, die mit großem Respekt anzuerkennen sind. Es ist auch mit Nachdruck festzuhalten, daß es damals wie heute immer wieder Weitsichtige gegeben hat, die auch im außerfachlichen Bereich ein Fortschreiten in menschlicher Aufgeschlossenheit möglich gemacht haben, die darum wußten, daß Medizin zwar grundlegend angewandte Naturwissenschaft sein muß, bei ihrer ärztlichen Tätigkeit, zumal im Gespräch, aber auch Kunst ist. Wir wollen uns klar sein, daß Blicke auf eine solche Vergangenheit nicht zum beruhigenden Gefühl veranlassen können, das sei ein passé défini. Sie sollten vielmehr durch Hinweise auf menschliche Problematik hellhöriger, verständnisvoller und kritischer machen für unsere Gegenwart und so auch zuversichtlicher für das Gehen neuer Wege in eine manchmal beschwerlich scheinende Zukunft.

Vielleicht hat Huizinga, der Leidener Geschichtsphilosoph, in seinem Buch «Im Banne der Geschichte» auch so etwas gemeint, wenn er Geschichte definiert hat als «die geistige Form, in der sich eine Kultur über ihre Vergangenheit Rechenschaft gibt».

Einen solchen kurzen Rechenschaftsbericht über eine nahe Vergangenheit wollte ich zu geben versuchen. Dabei ist mir klar, daß für manches, was sich erst vor recht kurzer Zeit zugetragen hat, die Distanz für ein sicheres Urteil wohl recht knapp ist, daß eine gewisse Subjektivität in der Auswahl der Probleme nicht zu vermeiden war. Nahe eigene zeitliche Präsenz am Geschehen mag das zum Teil ausgeglichen haben.

\section{Summary}

Some problematic aspects of the history of the faculty of medicine at the University of Basle between 1900 and 1933 are discussed. In 1900, seven of the eight medical professors in ordinary were Germans. Not every did feel at home here, and Basle was for him only a station to reach a position in his native country. Swiss candidates wanted, because the assistants were too badly paid. - The academic life was not free from antisemitism. The German Werner Gerlach, professor of pathology, was an engaged Nazi. But Basle had the good fortune that there lived eminent persons with a sober and far-sighted judgment.

Prof. Dr. med. Friedrich Rintelen, Bruderholzrain 55, CH-4059 Basel 